Available online on 15.01.2019 at http://jddtonline.info
(c) 2011-18, publisher and licensee JDDT, This is an Open Access article which permits unrestricted
non-commercial use, provided the original work is properly cited
Open Access to Pharmaceutical and Medical Research

Open $\odot$ Access

Research Article

\title{
Chitosan anchored nanoparticles for breast cancer: preparation and evaluation: part-I
}

\author{
Rajkumari Thagele, Archana Bagre and Mohan Lal Kori* \\ Vedica College of B. Pharmacy, RKDF University, Gandhi Nagar, Bhopal (M.P.) - India
}

\begin{abstract}
The objective of present research work was to develop methotrexate loaded chitosan anchored shell nanoparticles for drug delivery in breast cancer. Chitosan nanoparticles (CS-NPs) were synthesized by ionic gelation of chitosan using sodium tripolyphosphate (STPP). The optimized nanoparticles were characterized for particle size and polydispersity index (PDI) revealed particle size were found to be between $57.08 \mathrm{~nm}$ to169.5 $\mathrm{nm}$ and PDI 0.252 to 0.639 respectively. The results signpost that stirring speed during ionic gelation reaction was also decisive parameters for the size of the nanoparticles obtained. Further characterization involved to show polymer-drug interaction was FTIR and DSC. This paper grants a revision of the physical factors elaborate in attaining nanoparticles in order to regulate the particle size of polymeric nanoparticles made from chitosan, without any surplus chemical treatment.
\end{abstract}

Keywords: Breast cancer, Nanoparticles, Chitosan, Methotrexate

Article Info: Received 02 Nov 2018; Review Completed 11 Dec 2018; Accepted 18 Dec 2018; Available online 15 Jan 2019

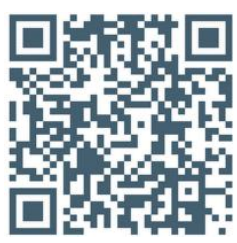

Cite this article as:

Thagele R, Bagre A, Kori ML, Chitosan anchored nanoparticles for breast cancer: preparation and evaluation: part-I, Journal of Drug Delivery and Therapeutics. 2019; 9(1):1-5 http://dx.doi.org/10.22270/jddt.v9i1.2151

*Address for Correspondence:

Mohan Lal Kori, Vedica College of B. Pharmacy, RKDF University, Gandhi Nagar, Bhopal (M.P.) - India

\section{INTRODUCTION}

In modern age of chemotherapy, cancer is one of the leading causes of the death all over the world. Cancer is state in which normal growth controlling mechanisms of the cells are permanently impaired, permitting the progressive growth of cells without reaching growth equilibrium. Since cancer cells proliferate rapidly, they need augmented supply of nutrients and this leads to over expression of various receptors on the surface of these cells. A basic tenet of pharmaceutical formulation science is that the dosage form exists to optimize the delivery of a pharmaceutical active to its site of action in the most effective and safe manner ${ }^{1}$.

Throughout previous epochs say 20 years, a generous amount of work has been conveyed on chitosan and its budding use in numerous bio applications. Chitosan is a linear polysaccharide composed of arbitrarily circulated $\beta$ (1-4)-linked d-glucosamine and N-acetyl-d glucosamine. As compared to various organic polymer chitosan have inherent properties i.e. non-toxicity, biocompatibility, biodegradability, cationic properties, bio adhesive characteristics and permeability-enhancing properties. Chitosan-constructed particles have been comprehensively deliberate for delivery of anti-cancer agents, therapeutic proteins, genes, antigens, and so on ${ }^{2-7}$. Chitosan is the most appropriate for custom as a non-viral vector for gene delivery, particularly with reverence to transfection efficiency ${ }^{8-9}$. Consequently, variability existing in the initial chitosan will undoubtedly govern the features of the nanoparticles obtained.

These nanoparticles can attain a size of up to $1000 \mathrm{~nm}$ and positive zeta potential of between $+20 \mathrm{mV}$ and $+60 \mathrm{mV}^{9-10}$ Chitosan-tripolyphosphate nanoparticles are envisioned for in-vivo administration, so that no detrimental effects exists that may jeopardies the welfare of the organism to which they are brought. The ionic gelation technique encounters this requirement; meanwhile the method does not require the toting of any organic solvents, thus evading the problematic exclusion of filtrates prior to conveyance into living organism ${ }^{11}$.

Breast cancer is the most common invasive cancer in women worldwide ${ }^{12}$. In cancer treatment, nanotechnology grasps great promise to transfigure drug delivery, gene therapy, diagnostics and many areas of research, development and clinical application ${ }^{13}$. In overall, nanocarriers may shield drug from degradation, enhance drug absorption by easing diffusion through epithelium, vary pharmacokinetic and tissue distribution profile, and/ or improve intracellular penetration. Also, Nano systems endured found valuable to improve the enactment of imaging techniques applied for the in vivo diagnosis of tumors ${ }^{14}$.

In recent years, a considerable work has been directed to the oral administration of anticancer drugs, on account of the 
need to facilitate drug administration in patients treated for cancer using a more acceptable route. The expectations are to improve convenience and to reduce hospitalization where possible.15-16The use of colloidal preparations could modify the biopharmaceutical properties of the drug, such as solubility and permeability, without changing its chemical structure.

A Nanoparticle is composed of atoms in either a single- or poly-crystalline arrangement having at least one dimension smaller than 100 nanometers ${ }^{17}$. Polymeric NPs have shown preferential accumulation at tumor sites, their usage as carriers improves efficacy and reduces side effects. Chitosan (CS) has been widely used to prepare nanoparticulate drug delivery system since has many good bio properties and physiochemical characteristics. CS is a natural polysaccharide which derived from chitin by deacetylation. This cationic polymer is regarded as biocompatible, biodegradable and non-toxic. The cationic properties of CS are particularly valuable for drug delivery systems. For example, ion complexes between CS and anionic drugs (i.e. methotrexate (MTX)) can be formed to NPs 18-20.

Ionotropic gelation technique is based on the ionic interactions between the positively charged $\mathrm{CH}$ and the negatively charged groups of polyanions, such as sodium tripolyphosphate, which is the most extensively, used ion crosslinking due to its non-toxic and multivalent properties ${ }^{2}$. Sodium tri polyphosphate is the only water soluble inorganic polyphosphate able to spontaneously form nanoparticles by electrostatic interactions between the long polyphosphate chains and the $\mathrm{CH}^{22-25}$.

Regarding the characteristic size range of chitosan nanoparticles, it is also of interest to analyses to what extent it is possible to obtain a higher percentage of a given particle size, depending on the mechanical parameters of the reaction. This information would contribute to defining a methodology for systematic and controlled production.

In this study we proposed to prepare, optimize and evaluate methotrexate loaded chitosan nanoparticles for targeted delivery towards breast cancer cells.

\section{MATERIALS AND METHODS}

\section{Materials:}

Methotrexate was received as gift sample from Khandelwal Laboratories Pvt. Ltd. Pune, India. Chitosan was obtained from Central Institute of Fisheries, Cochin; TPP and all other chemicals were AR grade and used as received.

\section{Preparation of carrier system:}

Chitosan nanoparticles were prepared according to a modified method of Calvo et al. 1996 12,26 with slight modifications based on the ionic gelation of chitosan with STPP anions. Chitosan (CH) solution $(2 \mathrm{mg} / \mathrm{ml})$ was prepared by dissolving $\mathrm{CH}$ in acetic acid solution (1.5\%) using magnetic stirrer (Aarson, India). Sodium tripolyphosphate solution $(1 \mathrm{mg} / \mathrm{ml})$ was added drop wise to $4 \mathrm{ml}$ at the rate of $1 \mathrm{ml} / \mathrm{min}$ to chitosan solution using syringe needle under vigorous magnetic stirring at room temperature. Sonicate the formulation for 15 minutes using sonicator (Digital Ultrasonic Cleaner, Jyoti Scientific, India), then disperse in water and separated by centrifugation (Rolex Centrifuge) for 10 minutes.

Various formulation and process variables i.e. weight ratio of chitosan and STPP, chitosan to enoxaparin weight ratio, stirring speed and stirring time, which affect characterization of nanoparticles, were optimized on the basis of their effect on drug entrapment efficiency and particle size. The various variables were optimized by varying one variable at a time and keeping other variables constant (Table 1). Based on an optimization procedure designed by us, a number of parameters were investigated by changing one parameter while keeping the others constant. The prepared nanoparticles were used for further studies.

\section{Characterization of nanoparticles}

Particle size and zeta potential determination: The average particle size and zeta potential of CS-NPs were determined by Photon correlation spectroscopy in a Zetasizer (DTS version 4.10, Malvern Instruments, England). Poly-disparity index (PDI) was also measured to determine particle size distribution.

Shape and Surface Morphology: Shape and surface morphology of nanoparticles was determined by scanning electron microscopy (SEM). For SEM, samples were examined under a scanning electron microscope (LEO 435 VP, Eindhoven Netherlands) at an acceleration voltage of 30 $\mathrm{kV}$, and photomicrographs were taken at $2890 \mathrm{X}$.

Fourier transform infrared spectroscopy study: The FTIR spectral measurement were carried out on a Perkin Elmer Spectrum 21- prestige spectrophotometer. The by triturating 3-5 mg of drug with polymer samples with 100-150 mg of $\mathrm{KBr}$ and compressed on a hydraulic press to make pellets. Spectra were taken by scanning the samples. The observed peaks were compared with those standard FTIR spectra. Indian Pharmacopoeia, 2010).

Differential Scanning Calorimetry study: DSC is a thermoanalytical technique used to observe fusion and crystallization events. Thermograms were obtained in a Shimadzu DSC-50 system (Shimadzu, Kyoto, Japan). 5 mg of lyophilized powder sample was placed in a standard aluminum pan and heated from 0 to $350{ }^{\circ} \mathrm{C}$ at a heating constant rate of $10 \circ \mathrm{C} / \mathrm{min}$ under constant purging of nitrogen at $20 \mathrm{~mL} / \mathrm{min}$.

\section{RESULTS AND DISCUSSION}

\section{Preparation and optimization:}

The characteristics of the chitosan/TPP particles prepared with different concentrations of chitosan or TPP were studied. The results indicated that the particle size increased with increasing the concentration of either chitosan or TPP. Calvo et al. found that the formation of chitosan/TPP nanoparticles was only possible for some specific concentrations of chitosan and TPP. This fact was also verified in our study that in order to avoid the formation of any micro-particles, the concentration of chitosan or TPP needed to be below $1.5 \mathrm{mg} / \mathrm{mL}$ and $1.0 \mathrm{mg} / \mathrm{mL}$, respectively. In these concentration ranges, it seemed that the concentration of chitosan or TPP had little effect on the monodispersity of the nanoparticles, since their PDI values were all below 0.05 . Results of optimization were illustrated in Table 1.

It is known that under acidic conditions, there is electrostatic repulsion between chitosan molecules due to the protonated amino groups of chitosan, meanwhile, there also exist inter chain hydrogen bonding interactions between chitosan molecules. Below a certain concentration of chitosan (2.0 $\mathrm{mg} / \mathrm{mL}$ as reported), the intermolecular hydrogen bonding attraction and the intermolecular electrostatic repulsion are in equilibrium ${ }^{27-29}$. Therefore, in this concentration range, as chitosan concentration increases, chitosan molecules approach each other with a limit, leading to a limited increase in intermolecular cross-linking, thus larger but still 
nanoscale particles are formed. Above this concentration, microparticles are easily formed probably due to the stronger hydrogen bonding interactions leading to plenty of chitosan molecules involved in the cross-linking of a single particle. The formation of micro-particles usually leads to a flocculent precipitate due to the electrostatic repulsion between particles are not sufficient to maintain the stability of these large particles.

It was also found that chitosan at low concentration could form stable nanoparticles even at a low mass ratio of chitosan to TPP, while chitosan at higher concentration could only form stable nanoparticles at a higher mass ratio of chitosan to TPP. For example, when the concentration of TPP was fixed at $0.5 \mathrm{mg} / \mathrm{mL}$, a chitosan concentration of 0.5 $\mathrm{mg} / \mathrm{mL}$ could form stable nanoparticles, while a chitosan concentration of $1.0 \mathrm{mg} / \mathrm{mL}$ would form aggregates. To explain this phenomenon, it is inferred that as chitosan concentration decreases, the intermolecular distance increases, thus leading to a decrease in intermolecular crosslinking between chitosan molecules while an increase in cross-linking density between chitosan and TPP, namely an increase in the ratio of moles of TPP to the moles of chitosan repeating units ${ }^{30}$.This can be utilized to prepare chitosan/TPP nanoparticles with smaller size, since an appropriate increase in the mass ratio is conducive to reduce the particle size.

\section{Characterization of carrier system:}

The optimized nanoparticles were characterized for particle size and polydispersity index (PDI) using Zetasizer Nano ZS90 (Malvern Instruments, Malvern, UK) showed particle size and PDI between $57.08 \mathrm{~nm}$ to $169.5 \mathrm{~nm}$ and 0.252 to 0.639 respectively (Fig 2). The results indicate that stirring speed during ionic gelation reaction is decisive for the size of the nanoparticles obtained.

\section{Shape and surface morphology:}

Prepared chitosan and alginate coated chitosan nanoparticles were characterized for shape and surface morphology, particle size, drug entrapment efficiency, zeta potential and polymer-polymer interaction. The shape and surface morphology of carrier system were characterized by SEM photomicrographs (Fig. 1). Nanoparticles were found to be spherical in shape but the surface of chitosan nanoparticles was found to be smooth.

\section{FTIR Analysis:}

Drug excipient interaction study was one of the important parameters which depict much information regarding the stability of formulations, drug release from them ${ }^{27}$.The FTIR spectral measurement were carried out on a Perkin Elmer Spectrophotometer. In order to examine the interaction between components of nanoparticulate systems, preliminary concerns were taken over comparative FTIR spectrum of drug MTX, chitosan, TPP and physical mixture of drug with chitosan and TPP shown in Fig 3. In MTX IR spectrum, peak at $3406 \mathrm{~cm}-1$ corresponds to $-0 \mathrm{H}$ group and $1600 \mathrm{~cm}-1$ corresponds to $\mathrm{C}=0$ band and the spectrum is comparable to that of earlier study. As shown in Fig.3 specific MTX peaks were observed in physical mixture.

\section{Differential Scanning Calorimetry (DSC):}

Drug excipient interaction study is used to get information about both the physical and the energetic properties of a compound. DSC is very useful for the investigation of the thermal properties of drug delivery carriers, providing both qualitative and quantitative information about the physiochemical state of drug inside the drug delivery system $^{26}$.There is detectable endotherm when the drug is present in molecular dispersion or solid solution in the polymeric system loaded with drug. ${ }^{27}$

Table 1: Data represents stirring time v/s particle size and polydispersity index (PDI) of different optimized formulations.

\begin{tabular}{|l|l|l|l|}
\hline Formulation Code & Stirring Time (hrs) & Particle size (nm) & PDI \\
\hline R1S1 & 0.5 & $194.33 \pm 8.1$ & $0.519 \pm 0.021$ \\
\hline R2S2 & 1.0 & $84.30 \pm 3.3$ & $0.254 \pm 0.011$ \\
\hline R3S3 & 1.5 & $56.37 \pm 2.4$ & $0.212 \pm 0.009$ \\
\hline R4S4 & 2.0 & $91.4 \pm 4.4$ & $0.250 \pm 0.013$ \\
\hline R5S5 & 2.5 & $168.1 \pm 9.2$ & $0.637 \pm 0.021$ \\
\hline
\end{tabular}

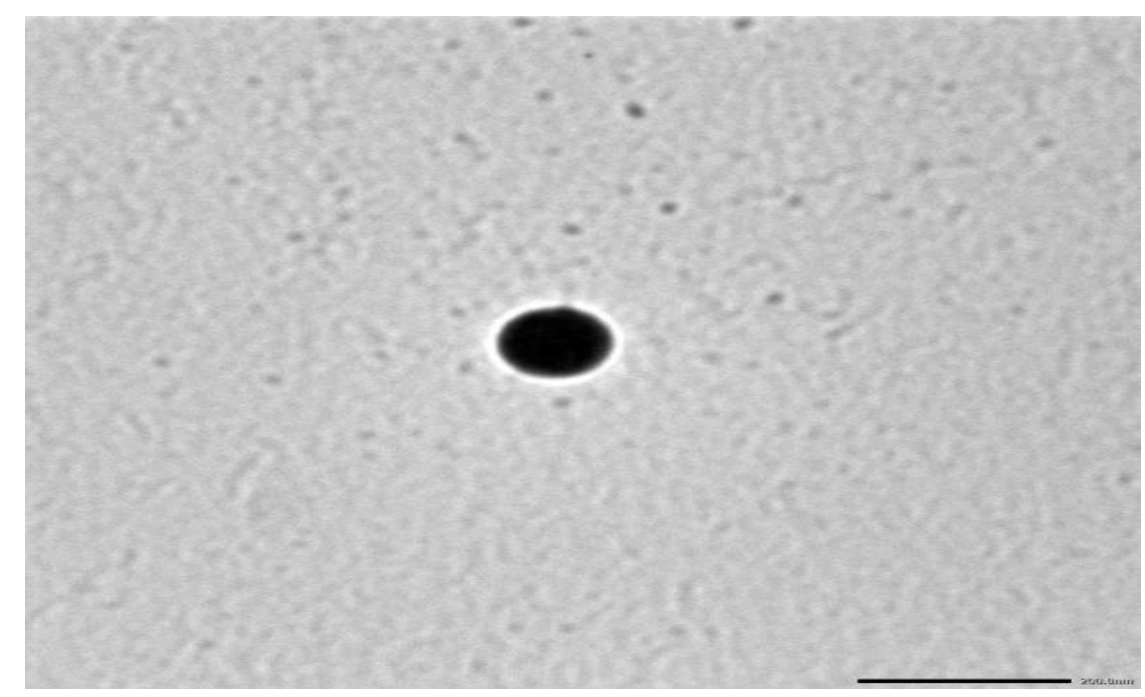

Figure 1: Photomicrograph of chitosan nanoparticles by SEM analysis. 




Figure 2: particle size distribution of chitosan nanoparticles

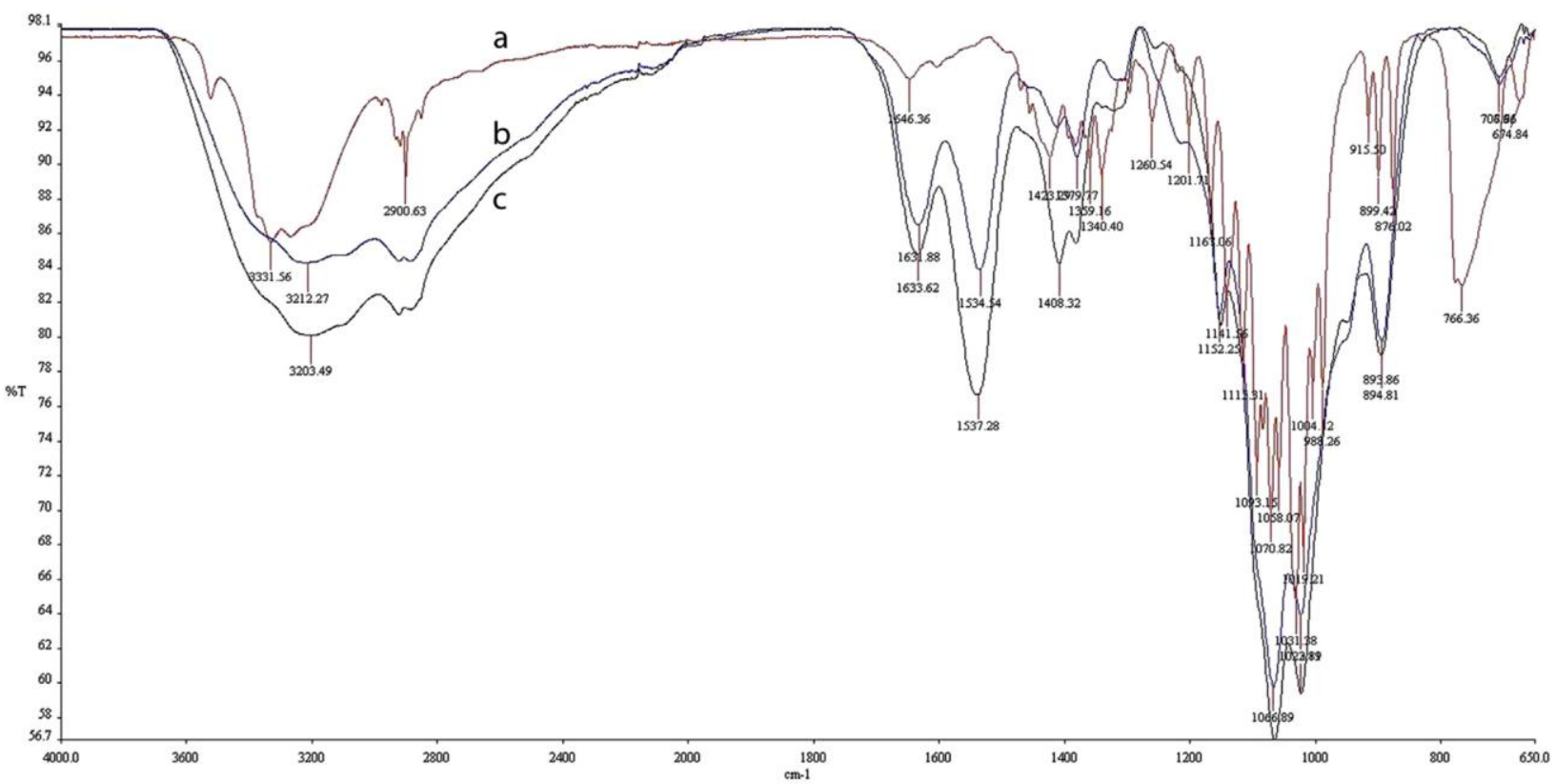

Figure 3: FTIR spectra of MTX, CS, TPP and physical mixture of MTX,CS and TPP

\section{CONCLUSION}

Chitosan anchored nanoparticles of MTX were prepared by ionotropic gelation method. The polymeric particles in a nanosize range with a desired drug polymer ratio can be obtained. It may be a potential alternative dosage form of the drug for the treatment of breast cancer.

\section{ACKNOWLEDGEMENTS}

Authors are thankful to Khandelwal Labs. Pvt. Ltd. Pune, India for generously supplying MTX. Central Institute of Fisheries and Technology, Pune, India for providing chitosan. SAIL, SOPS, RGPV, Bhopal (M.P.) for carrying PCS and FTIR and DSC study and UGC for providing grant Rajiv Gandhi National Fellowship for SC to carry out the research work. 


\section{REFERENCES}

1. Phulzalke SB, Kate BA, Bagade MY, Shete RV, Formulation Development and Evaluation of Orodispersible Tablets of Quetiapine Fumarate by Sublimation Method, Asian Journal of Biomedical and Pharmaceutical Sciences, 2016; 6(57):22-31.

2. Min Li, Ge Li, Lu Tang, Lu Xingyan, Jun Chen, Li Qingnan, Guozhu Yang, Huanhuan Jia, Wu Yu'e, Yu Zhang, Tumor Necrosis Factor (TNF-A)-Induced Upregulation of Cartilage Degradation-Associated Genes by Chondrocytes Requires Epithelial Sodium Channel Activity Asian J Biomed Pharmaceut Sci 2017; 7(6).

3. Kumari A, Yadav SK, Yadav SC, Biodegradable polymeric nanoparticles based drug delivery systems, Colloids and Surfaces B: Biointerfaces; 2010; 72:1-18.

4. Tripathi CB, Parashar P, Arya M, Singh m, Kanoujia J, Kaithwas G, Saraf SA, QbD-based development of $\alpha$-linolenic acid potentiated nanoemulsion for targeted delivery of doxorubicin in DMBA-induced mammary gland carcinoma: in vitro and in vivo evaluation, Drug Delivery and Translational Research; 2018,8(5):1313-1334.

5. Heineman TE, Sabbas A, Delamerced MS, Y. Chiu, M. Smith, Parashar B, Wernicke AG, Impact of a large breast separation on radiation dose delivery to the ipsilateral lung as result of respiratory motion quantified using free breathing and 4D CT-based planning in patients with locally advanced breast cancers: A potential for adverse clinical implications, Journal of CANCER Research and Therapeutics;2013; 9(1):154160.

6. Krishnaveni B. Chitosan: A review on its varied novel therapeutic and industrial applications. Journal of Drug Delivery and Therapeutics, 2016; 6(6):70-79. https://doi.org/10.22270/jddt.v6i6.1328

7. Kaur M., Bhatia A., Sethi D., Kaur G., Vig K. Hypoglycemic potential of probiotic DNA loaded chitosan nanoparticles: an in vivo study. Journal of Drug Delivery and Therapeutics, 2017; 7(2):70-76. https://doi.org/10.22270/jddt.v7i2.1405

8. Wang JJ, Zeng ZW, Xiao RZ, Xie T, Zhou GL, Zhan XR, Wang SL, Recent advances of chitosan nanoparticles as drug carriers, Int. J. Nano.; 2011; 6:765-774.

9. Sinha VR, Singla AK, Wadhawan S, Kaushik R, Kumria R, Bansal $\mathrm{K}$, Dhawan S, Chitosan microspheres as a potential carrier for drugs, Int. J. Pharma.;2004; 274:1-33.

10. Singla AK, Chawla M, J. Pharm. Pharmacol; 2001; 53:1067.

11. Kong F, Liu G, Sun B, S. Zhou, A. Zuo, R. Zhao, D. Liang, Phosphorylatable short peptide conjugated low molecular weight chitosan for efficient siRNA delivery and target gene silencing, Int. J. Pharm.; 2012; 422:445-453.

12. Calvo P, Remunan-Lopez C, Vila-Jato JL, Alonso ML, Novel hydrophilic chitosan-polyethylene oxide nanoparticles as protein carriers,J. Appl. Polym. Sci.; 1997; 63:125-132.

13. Csaba N, Koping-Hoggard M, Alonso MJ, Ionically crosslinked chitosan/ tripolyphosphate nanoparticles for oligonucleotide and plasmid DNA delivery,Int. J. Pharm.; 2009; 382:205-214.

14. Dash, F. Chiellini, R.M. Ottenbrite, E. Chiellini, Chitosan - a versatile semisynthetic polymer in biomedical applications, Prog. Polym. Sci.; 2011; 36:981-1014.

15. H. Wang, Y. Zhao, H. Wang, J. Gong, H. He, M.C. Shin, V.C. Yang, Y. Huang, Lowmolecular-weight protamine-modified PLGA nanoparticles for overcoming drug-resistant breast cancer, J. Control. Rel.; 2014; 192:47-56.

16. H. Hong, Y. Zhang, J. Sun, W. Cai, Molecular imaging and therapy of cancer with radiolabeled nanoparticles, Nano Today; 2004; $4(5): 399-413$

17. Nikita Sehgal, Kriti Soni, Navika Gupta, Kanchan Kohli, Microorganism Assisted Synthesis of Gold Nanoparticles: A Review Asian J Biomed Pharmaceut Sci. 2018; 8(64).

18. P. Couvreur, C. Vauthier, Nanotechnology: intelligent design to treat complex disease, Pharm. Res.; 2006; 23:1417-1450.

19. M.D. DeMario and M.J. Ratain. Oral chemotherapy: rationale and future directions, J. Clin.Oncol.; 1998; 16:2557-2567.

20. A. Sparreboom, M.J. de Jonge, J.Verweij, The use of oral cytotoxic and cytostatic drugs in cancer treatment, Eur. J. Cancer.; 2002, 38(1):18-22.

21. Y. Wang, X. Yang, J. Yang, Y. Wang, R. Chen, J. Wu, Y. Liu, N. Zhang, Selfassembled nanoparticles of methotrexate conjugated 0-carboxymethyl chitosan: preparation, characterization and drug release behavior in-vitro, Carbohydr. Polym.; 2011; 86:1665-1670.

22. C. Gao, T. Liu, Y. Dang, Z. Yu, W. Wang, J. Guo, X. Zhang, G. He, H. Zheng, Y. YX. Kong, $\mathrm{pH} /$ redox responsive core cross-linked nanoparticles from thiolated carboxymethyl chitosan for in vitro release study of methotrexate, Carbohydr. Polym.; 2014, 964-970.

23. J. Lu, Y. Pang, F. Xie, H. Guo, Y. Li, Z. Yang, X. Wang, Synthesis and in vitro/ in vivo evaluation of $99 \mathrm{mTc}$-labeled folate conjugates for folate receptor imaging, Nucl. Med. Biol.; 2011; 38(4):557-565.

24. W. Fan, W. Yan, Z. Xu and H. Ni, Formation mechanism of monodisperse, low molecular weight chitosan nanoparticles by ionic gelation technique. Colloids and Surfaces B: Biointerfaces; 2012; 90:21-27.

25. L. Casettari, M. Cespi, G.F. Palmieri and G. Bonacucina, Characterization of the interaction between chitosan and inorganic sodium phosphates by means of rheological and optical microscopy studies. Carbohyd. Pol.; 2013; 91(2):597602.

26. D.M. Pickup, R.J. Newport, E. R. Barney, J. Y. Kim, S. P. Valappil and J. C. Knowles, Characterisation of phosphate coacervates for potential biomedical applications, J Biomaterials Appli.;2014; 28(8):1226-1234.

27. M. Muller, M. Brunner, R. Schmid, M. Robert Mader, J. Bockenheimer, G. Steger, G. Steiner, B., Eichler, B. B. Daum, Interstitial Methotrexate Kinetics in Primary Breast Cancer. Advances in Brief Cancer Res.; 1998; 58:2982-2985.

28. A. Jain and S. K. Jain, In vitro and cell uptake studies for targeting of ligand anchored nanoparticles for colon tumors, Europ.J.Pharm.Sci.;2008; 35(5):404-416.

29. R.S Nair, Preeti Bisht, Sarangi TK, Insights on Cancer Preventive Approaches Using Plant Based Extracts Asian J Biomed Pharmaceut Sci 2018; 8(65).

30. B. G. Gowda, Mallappa M, R.T. Mahesh, C. C. Hadimani, Spectroscopic and Viscositic Studies on the Interaction of Solifenacin Succinate with DNA Asian Journal of Biomedical and Pharmaceutical Sciences; 2014; 4(35):44-48. 